\title{
Research and Development of Visual Systems Software in Automobile Driving Training Simulator
}

\author{
Zhenzhen Fan \\ Automotive Engineering Institute, Jiangxi University of Technology, Nanchang 330098, China
}

\begin{abstract}
In China, with the rapid economic development, the speed driving, dense vehicles on the road, family-oriented car and amateurism of drivers all have become an inevitable trend. However, due to China's large population, the transport facilities situation is also unsatisfactory, it is relatively difficult to drive, and the probability of traffic accidents is also great, and especially the new drivers account for the greater proportion of accidents. Real training of drivers needs to consume expensive oil, plus a large number of vehicle maintenance costs; moreover, the damage to the ecological environment such as noise, emissions and other pollution caused by the new driver are much higher than a skilled driver. These problems are very real and urgent. The use of computer technology, control technology, sound technology, mechanical engineering technology, vehicle engineering technology and a variety of real vehicle controls and instrument panel all make a realistic simulation of vehicle environment and the cockpit environment, in which trainees can drive with vehicle true feeling. This paper focuses on visual system of automobile driving training simulator, and studies the establishment of model as well as the display technology of multi-channel graphics, in particular the study of computer display frame depth graphics; the paper tries to solve the problems when the image display speed and time are inconsistent to the actual vehicle speed and time.
\end{abstract}

\section{Introduction}

The traditional driving training usually consists of theories learning and driving technology. Theoretical training enables trainees to understand the structure of the vehicle, traffic rules and safe driving, etc.; driving skills training enables trainees to operate the vehicle, grasp the essentials of driving skills under various environments under the guidance of coach. Such step by step traditional training method is too rigid and inflexible, especially when it is a society emphasizing time and efficiency, and it is obviously not suitable to a lot of non-professional driver and driving training with special demand. Automobile driving training simulator is the most effective training tool, not only improving the level of driving skill, but also reducing the various fees. Therefore, it is quite in line with China's national conditions, and has significant social and economic significance to study and develop the vehicle driving training simulators that are suitable for China's road conditions and traffic regulations, driver training program and curriculum, and to put it into wide use. This will not only greatly improve the quality and the efficiency of training on the driver, but also promote the training to take on modern, standardized and scientific way.

\section{Classification of Automobile Driving Training Simulator}

Depending on different visual systems, automobile driving training simulators can be divided into two types: active and passive. Active automobile driving training simulator utilizes vehicle dynamics, 
kinematics, computer technology with the support by electronic, hydraulic and control technology, which can be used as initial training for the driver; in addition to a visual screen, vehicle drum driving simulator also places simulators on the drum for analog driving, producing a certain degree of road feelings, sound and movement sense; however, it cannot simulate acceleration and deceleration, cornering and other conditions. Therefore, it has a lower fidelity simulation; a movable seat simulator driving simulator is overall supported by a plurality of hydraulic cylinders, through the electronically - controlled hydraulic system, the simulator simulates movement conditions and attitude of vehicle on the road, so as to provide a more realistic vision auditory, tactile and somatosensory simulation for the driver .

Visual system simulation applies visual simulation technology for a variety of simulators visual system, in order to produce as much as possible the effect of the real scene; it has comprehensively utilized computer graphics, information theory, computer vision, image processing, pattern recognition, software engineering theory and application technology.

\section{System Structure of Automobile Driving Training Simulator}

It has used the following design, that is, from the perspective of ergonomics, through a variety of sensors and external input to constitute a feedback closed loop, and achieve the system real-time response to external input via link communication, so as to complete real feeling simulation of driving. According to the development of ideas and design principles proposed, this automobile driving training simulator studies by paper is using the system structure shown in Fig. 1.

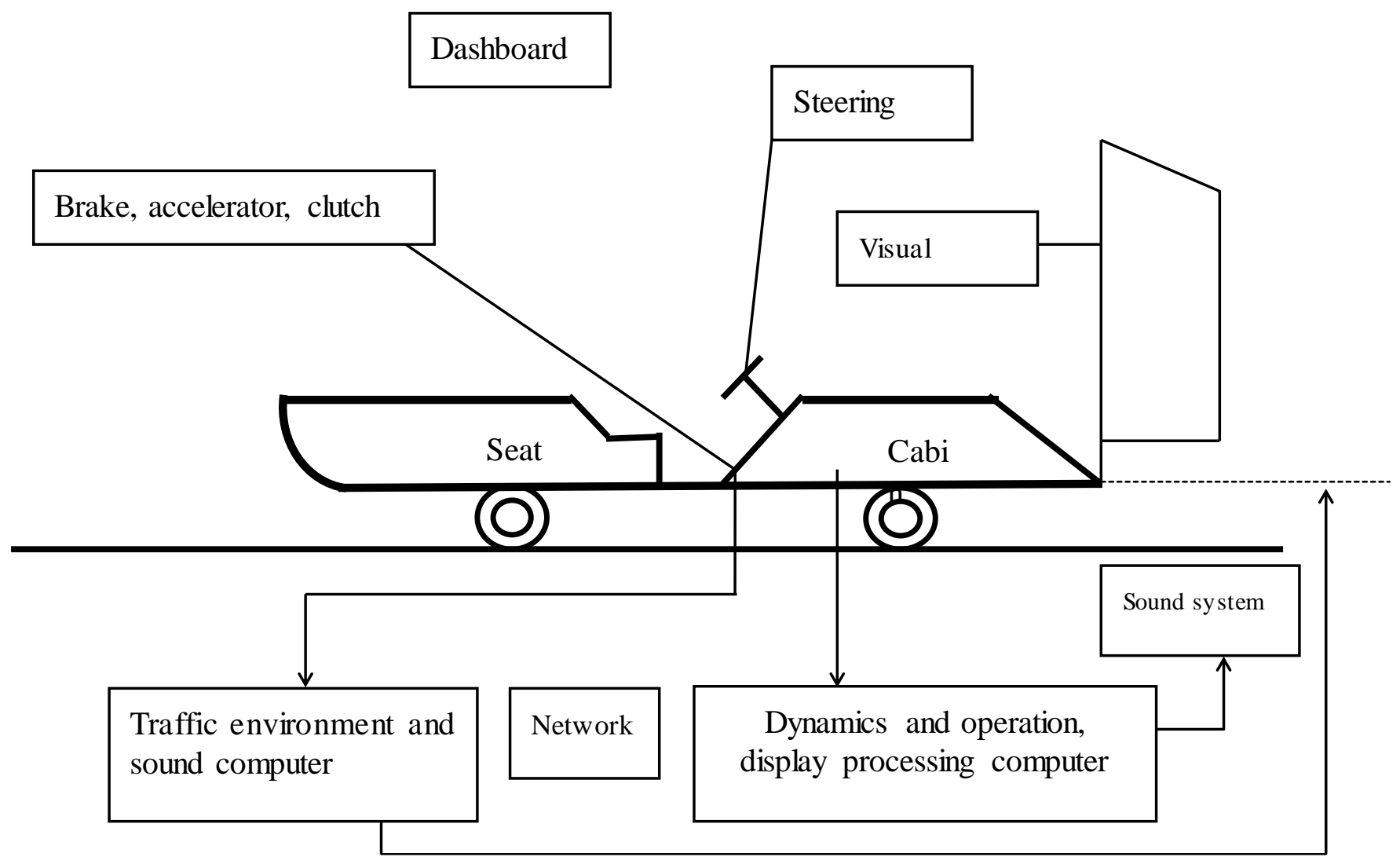

Fig. 1 System Structure of Automobile Driving Training Simulator

As for bi-axial vehicle, from studies conducted in the past, two degrees of freedom linear car model has been used before. During preliminary analysis on automobile curve, the automobile is 
seen as parallel to the road. i.e., there is not vertical movement, and displacement along the vertical axis is zero; transverse plane pitch angle about perpendicular to the vehicle longitudinal plane , the roll angle around the forward direction axis of the vehicle is zero. Thus, the vehicle only has two degrees of freedom: lateral movement of the transverse plane perpendicular to the vehicle longitudinal axis and the yawing motion around the vertical axis.

As shown in Fig. 2 , take a relative coordinate system fixed to the vehicle, the origin 0 is intersection of the vertical line of gravity when the vehicle is stationary and the roll axis (front roll center wiring); the vehicle horizontal longitudinal axis is $\mathrm{X}$-axis; the automobile forward direction is positive. On the plane where the $\mathrm{X}$-axis lies, perpendicular to the $\mathrm{X}$-axis through the origin 0 is $\mathrm{Y}$ axis. Facing automotive forward direction, the left direction is positive.

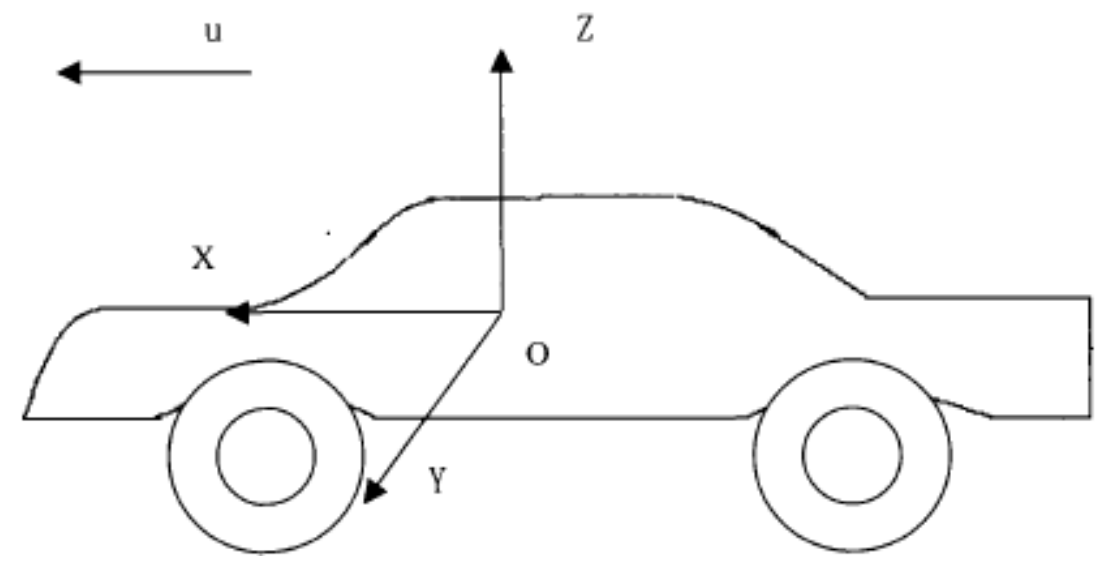

Fig. 2 Automobile Coordinate System

In the established relative coordinate system, vehicle longitudinal acceleration is:

$$
\dot{u}-v r
$$

Thus, the inertial force along the $\mathrm{X}$ axis is:

$$
\mathrm{M}(\dot{u}-v r)
$$

Automobile driving force

Here the main consideration is the force acting on the drive wheels when the engine torque transmitted through the driveline torque, while ignoring the impact of rolling resistance, air resistance, etc., so the driving force of the automobile when traveling is:

$$
T_{\omega} i \eta / R
$$

In the established relative coordinate system, projection of the absolute acceleration of the coordinates origin $\mathrm{O}$ in the $\mathrm{Y}$-axis direction is:

$$
\ddot{Y}=u \frac{d \varphi}{d t} \cos \beta
$$

The projection of lateral absolute acceleration of sprung mass gravity center on the $\mathrm{Y}$ axis is:

$$
\ddot{Y_{S}}=\ddot{Y}+h \dot{p}=u(r+\dot{\beta})+h \dot{p}=(u r+\dot{V})+h \dot{p}
$$

Automobile Differential Equation

According to D'Alembert's principle dynamic and static methods, we can list the force and torque balance equation of automobile five degrees of freedom model. 
Movement force balance equation along the $\mathrm{X}$-axis:

$$
\mathrm{M}(\dot{u}-v r)=T_{\omega} i \eta / R
$$

In addition, from the geometric relationship we have:

$$
\left\{\begin{array}{c}
\delta_{1}=\beta+\frac{a}{u} r-E_{f} \phi-\delta \\
\delta_{2}=\beta-\frac{b}{u} r-E_{f} r \\
\vartheta=i \bullet \delta
\end{array}\right.
$$

From the tire characteristics, we can see:

$$
\left\{\begin{array}{l}
P_{y 1}=2 k_{f} \delta_{1} \\
P_{y 2}=2 k_{r} \delta_{2}
\end{array}\right.
$$

Based on the numerical solution of differential equations integration ---- fourth-order Runge-Kutta method, we can solve differential equations above.

\section{Vision System Model}

Realistic Rendering (namely the establishment of the visual system model) is an integral part of the vehicle driving training simulator. Only the photorealistic graphics could reflect the precise meaning of "virtual". Realistic Rendering technology has utilized mathe matics, physics, computer graphics and other scientific knowledge, and generated a realistic graphics on computer graphics equipment as color photos. Based on the above work, it will finalize the establishment of the entire vehicle driving training simulator visual systems model.

The whole scene model of automobiles driving training simulator visual systems is made of 3D and 2D models; according to different functions and locations of these models, they are arranged in a certain order and formed virtual environment as driving training desired. Automobile driving training simulator visual systems have used modeling tools of MulitGenrCeaotrV2.4 scene models, from the performance requirements of the various materials, the process of scene model building is divided into five stages. Specific work-flow model is shown in Fig. 3. 


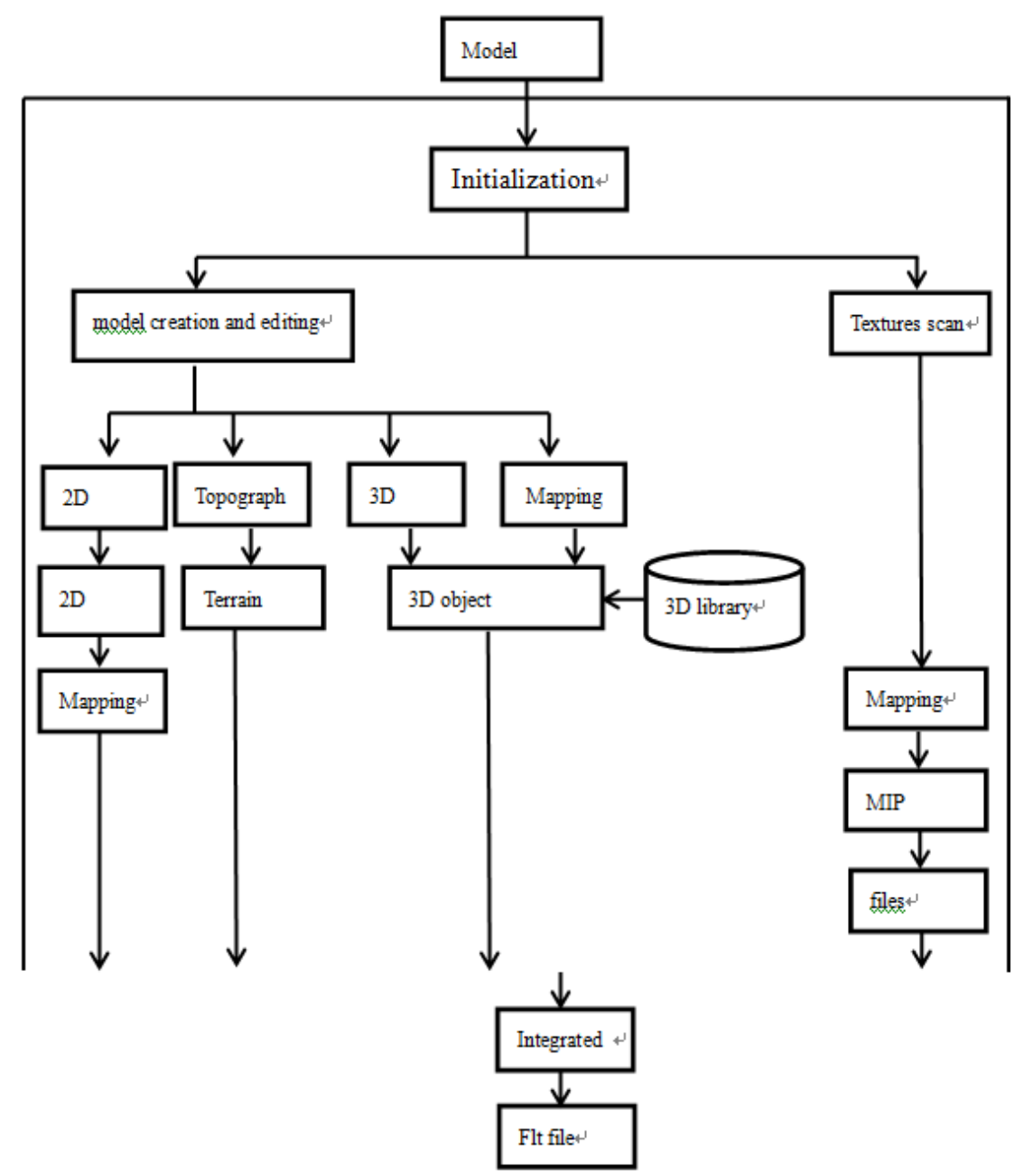

Fig. 3 Flow Chart of Automobiles Driving Training Simulator Visual System Modeling

\section{Conclusions}

The speed driving, dense vehicles on the road, pedestrian \& vehicle mixing traffic are the basic characteristics of the transport industry in China; while, with the growth of the national economy, people's living standards have been improving, the car becomes family oriented, hence the components of the drivers become more complex, which makes non-professional drivers become a more significant new feature of China's transportation industry. As for research and development of automobiles driver training simulator, this paper has completed the following work:

1. Studied the consistent match between speed and time of image change and speed and time running of the actual vehicle, and verified this method by simulation and experiment. The results showed: on the road of the same length, the speed of and time image motion are consistent with that of the actual cars.

2. In the paper, the building process of visual system scenario and implementation method of multi-channel technology could be applied not only to automobiles driving training simulator visual systems software development, but also to flight simulators, tank simulators and virtual battlefield scene establishment and implementation. 
Prospect and future of the automobiles driver training simulator: in order to achieve real purpose of training drivers, as well as developing a new type of automobiles driving training simulator under the existing conditions, we shall take the following technical ways:

1. Choose acceptable high performance and low cost hardware platform, and develop the advanced software development tools, so as to create a richer, vivid scene.

2. To further improve the real-time interaction of graphics rendering.

In summary, for the automotive driving training simulator, the current technology is at a turning point. The introduction of new technical means, which improves the performance of automotive driving training simulator, but also maintains the user price acceptability the price -- it is bound to promote the improvement in the level of driving training and traffic conditions.

\section{Acknowledgements}

This work was financially supported by the key subject building project (vehicle engineering) of Jiangxi University of Technology.

\section{References}

[1] Kuhl J, Evans D, Papelis Y, et al. The Iowa Driving Simulator: an immersive research environment[J]. Computer, 1995, 28(7): 35-41.

[2] Freeman J S, Watson G, Papelis Y E, et al. The Iowa driving simulator: An implementation and application overview[R]. SAE Technical Paper, 1995.

[3] Weir D H, Clark A J. A survey of mid-level driving simulators[R]. SAE Technical Paper, 1995.

[4] Reymond G, Kemeny A, Droulez J, et al. Role of lateral acceleration in curve driving: Driver model and experiments on a real vehicle and a driving simulator[J]. Human Factors: The Journal of the Human Factors and Ergonomics Society, 2001, 43(3): 483-495.

[5] Ball K, Owsley C, Sloane M E, et al. Visual attention problems as a predictor of vehicle crashes in older drivers[J]. Investigative ophthalmology \& visual science, 1993, 34(11): 3110-3123.

[6] Drosdol J, Panik F. The Daimler-Benz driving simulator a tool for vehicle development[R]. SAE Technical Paper, 1985.

[7] Frank L H, Casali J G, Wierwille W W. Effects of visual display and motion system delays on operator performance and uneasiness in a driving simulator[J]. Human Factors: The Journal of the Human Factors and Ergonomics Society, 1988, 30(2): 201-217.

[8] Thomke S H. Simulation, learning and R\&D performance: Evidence from automotive development[J]. Research Policy, 1998, 27(1): 55-74.

[9] Alluisi E A. The development of technology for collective training: SIMNET, a case history[J]. Human Factors: The Journal of the Human Factors and Ergonomics Society, 1991, 33(3): 343-362.

[10]Land S M, Hannafin M J. A conceptual framework for the development of theories-in-action with open-ended learning environments[J]. Educational Technology Research and Development, 1996, 44(3): 37-53. 